\title{
Systematic Subsegmentectomy by Ultrasound-Guided Finger Compression for Hepatocellular Carcinoma in Cirrhosis
}

\author{
Guido Torzilli, MD, PhD, Matteo Donadon, MD, Matteo Cimino, MD, Daniele Del Fabbro, MD, \\ Fabio Procopio, MD, and Florin Botea, MD
}

Liver Surgery Unit, Third Department of Surgery, University of Milan School of Medicine, IRCCS Istituto Clinico Humanitas, Rozzano, Milan, Italy

\begin{abstract}
Background. Systematic and extensive use of intraoperative ultrasound (IOUS) allows us to perform new conservative surgical procedures in liver surgery. This video shows systematic subsegmentectomy by IOUS-guided finger compression for a case of hepatocellular carcinoma (HCC) in cirrhosis.

Methods. The case of a 69-year-old woman with a single $3.5-\mathrm{cm} \mathrm{HCC}$ in segment 3 is presented. The patient has hepatitis $\mathrm{C}$ virus (HCV)-related well-compensated cirrhosis. After performing a T-inverted laparotomy, the IOUS is carried out for staging. Then, the area of resection is anatomically marked by IOUS-guided finger compression of
\end{abstract}

the subsegmental portal branch feeding the tumor in segment 3. Thus, the resection is performed under intermittent Pringle maneuver using Pean-clasia and bipolar forceps.

Results. Ninety-day mortality and morbidity for this patient were nil. No blood transfusions were required. The patient was discharged 8 days after surgery.

Conclusions. Systematic subsegmentectomy by IOUSguided finger compression is a feasible and effective technique, especially for HCC in cirrhosis. It may be potentially applied in each segment of liver as long as the thickness of the parenchyma and the anatomy of liver are suitable. We believe that this technique should be part of the modern liver surgeon's armamentarium.

Electronic supplementary material The online version of this article (doi:10.1245/s10434-009-0457-x) contains supplementary material, which is available to authorized users.

(C) Society of Surgical Oncology 2009

First Received: 23 January 2009;

Published Online: 30 April 2009

G. Torzilli, MD, $\mathrm{PhD}$

e-mail: guido.torzilli@unimi.it 